\title{
QTL mapping and transcriptome analysis identify candidate genes regulating pericarp thickness in sweet corn
}

\author{
Xiaming Wu ${ }^{1,2}$, Bo Wang ${ }^{1,2}$, Fugui Xie ${ }^{1,2}$, Liping Zhang ${ }^{1,2}$, Jie Gong ${ }^{1,2}$, Wei Zhu ${ }^{1,2}$, Xiaoqin Li ${ }^{1,2}$,
} Faqiang Feng ${ }^{1,2^{*}}$ and Jun Huang ${ }^{1,2^{*}}$ (D)

\begin{abstract}
Background: In recent years, the planting area of sweet corn in China has expanded rapidly. Some new varieties with high yields and good adaptabilities have emerged. However, the improvement of edible quality traits, especially through the development of varieties with thin pericarp thickness, has not been achieved to date. Pericarp thickness is a complex trait that is the key factor determining the edible quality of sweet corn. Genetic mapping combined with transcriptome analysis was used to identify candidate genes controlling pericarp thickness.
\end{abstract}

Results: To identify novel quantitative trait loci (QTLs) for pericarp thickness, a sweet corn $\mathrm{BC}_{4} \mathrm{~F}_{3}$ population of 148 lines was developed using the two sweet corn lines M03 (recurrent parent) and M08 (donor parent). Additionally, a high-density genetic linkage map containing 3876 specific length amplified fragment (SLAF) tags was constructed and used for mapping QTLs for pericarp thickness. Interestingly, 14 QTLs for pericarp thickness were detected, and one stable QTL (qPT10-5) was detected across multiple years, which explained 7.78-35.38\% of the phenotypic variation located on chromosome $10(144,631,242-145,532,401)$. Forty-two candidate genes were found within the target region of aPT10-5. Moreover, of these 42 genes, five genes (GRMZM2G143402, GRMZM2G143389, GRMZM2G143352, GRMZM6G287947, and AC234202.1_FG004) were differentially expressed between the two parents, as revealed by transcriptome analysis. According to the gene annotation information, three genes might be considered candidates for pericarp thickness. GRMZM2G143352 and GRMZM2G143402 have been annotated as AUX/ IAA transcription factor and ZIM transcription factor, respectively, while GRMZM2G143389 has been annotated as FATTY ACID EXPORT 2, chloroplastic.

Conclusions: This study identified a major QTL and candidate genes that could accelerate breeding for the thin pericarp thickness variety of sweet corn, and these results established the basis for map-based cloning and further functional research.

Keywords: Sweet corn, Pericarp thickness, QTL, Transcriptome, SLAF tags

\footnotetext{
* Correspondence: fengfa@scau.edu.cn; junhuang@scau.edu.cn

'The State Key Laboratory for Conservation and Utilization of Subtropical

Agro-bioresources, South China Agricultural University, Guangzhou 510642, Guangdong, People's Republic of China

Full list of author information is available at the end of the article
}

(c) The Author(s). 2020 Open Access This article is licensed under a Creative Commons Attribution 4.0 International License, which permits use, sharing, adaptation, distribution and reproduction in any medium or format, as long as you give appropriate credit to the original author(s) and the source, provide a link to the Creative Commons licence, and indicate if changes were made. The images or other third party material in this article are included in the article's Creative Commons licence, unless indicated otherwise in a credit line to the material. If material is not included in the article's Creative Commons licence and your intended use is not permitted by statutory regulation or exceeds the permitted use, you will need to obtain permission directly from the copyright holder. To view a copy of this licence, visit http://creativecommons.org/licenses/by/4.0/ The Creative Commons Public Domain Dedication waiver (http://creativecommons.org/publicdomain/zero/1.0/) applies to the data made available in this article, unless otherwise stated in a credit line to the data. 


\section{Background}

Sweet corn is a maize-derived vegetable crop developed through one or several recessive endosperm mutations that reduce the synthesis of starch and increase the accumulation of sugars or other short-chain polysaccharides. The growing area of sweet corn has increased rapidly in China. In recent years, some new varieties with high yields and good adaptabilities have emerged. However, improvement of edible quality traits, especially through the development of varieties with reduced pericarp thickness, has not been achieved to date. Kernel tenderness, crispness and residue percentage of sweet corn are important criteria for evaluating edible quality. Pericarp thickness and structure are closely related to these three quality traits. Thin pericarps show high softness, high crispness, low residue rate, and good taste [1]. The pericarp of corn is composed of nondigestible cellulose, which is neither nutritious nor digestible, and the thickness of the pericarp affects the tenderness of sweet corn. Thus, the selection of a thinner pericarp is highly important in sweet corn quality breeding programs. Reducing pericarp thickness has become an important breeding goal to improve the edible quality of sweet corn.

The genetic characteristics of maize pericarp thickness have a high narrow sense heritability $(55-82 \%)$ and involve additive effects, dominant effects and significant epistasis effects [2-4]. The number of effective genes associated with pericarp thickness estimates averaged 3.3 and ranged from 1.4 to 5.9 for the eight crosses [4]. To date, no single gene with a major effect $(>25 \%)$ has been identified. Haddad showed that the cell layer number of maize hybrids was the same as that of their female parents, and the difference in pericarp thickness was due to the thickening of the cell wall of the hybrids, which indicated that the thickness of the maize pericarp was affected by both maternal and nuclear genes [5]. Tracy and Schmidt analysed the pericarp thickness of 7 different sweet corn near-isogenic lines that differed in endosperm composition [sugary (su), dull $(d u)$, waxy $(w x)$, sugary-2 (su2), and shrunken-2 (sh2)] using a pressure micrometer and found that the pericarp thickness was significantly affected by embryo type, endosperm type, endosperm by inbred interactions, ear, and position of measurement on the kernel [6]. A study by Helm and Zuber showed that the narrow heritability of pericarp thickness was 0.80 and that pericarp thickness was controlled by 3 to 8 semidominant, epistatic and additive genes [2]. ITO and Brewbaker showed that pericarp thickness was controlled by $2-5$ semidominant genes [4]. Therefore, the gene loci and their genetic effects controlling pericarp thickness may be more easily identified and analysed by molecular methods.

To the best of our knowledge, few studies on linkage mapping for maize pericarp thickness have been reported.
For example, eight chromosomal fragments affecting sweet corn pericarp thickness were identified using restriction fragment length polymorphism (RFLP) markers in a chromosome segment substitute lines (CSSL) population [7]. Wang and Brewbaker used 94 maize recombinant inbred lines and identified three pericarp thickness QTLs located on chromosomes 1, 2 and 6 [8]. Forty-one QTLs related to pericarp thickness were detected using 100 simple sequence repeat (SSR) markers. Moreover, principal component analysis indicated that the first principal component composed of 8 QTLs could explain $87.60 \%$ of pericarp thickness phenotypic variance, which could be applied in breeding thin pericarp maize varieties $[9,10]$. Choe and Rocheford TR also found that some QTLs controlling pericarp thickness in a waxy corn mapping panel were colocated with ear trait QTLs, which may be due to the high correlation between pericarp thickness and ear traits [11]. Eight pericarp thickness QTLs were detected based on two genetic models using the $190 \mathrm{BC}_{1} \mathrm{~F}_{2}$ population. Three QTLs for pericarp thickness were identified using the composite interval mapping (CIM) method and explained 8.6, 16.0 , and $7.2 \%$ of phenotypic variation, respectively. Based on the mixed model-based CIM (MCIM) method, five QTLs for pericarp thickness were detected [12]. Although these investigations have been undertaken, no pericarp thickness genes have been cloned to date. These genetic maps commonly have low marker density, which makes it difficult to cover the whole genome, thereby making QTL analysis difficult. Next-generation sequencing (NGS) technology makes it possible to detect large quantities of SNP markers covering the entire genome. Specific length amplified fragment sequencing (SLAF-seq), first developed by Sun et al. [13], has been widely used for high-density genetic map construction, such as cotton [14], grape [15], and cucumber [16]. Compared with traditional molecular markers, the distribution density of markers in genetic mapping affects the accuracy of the mapping, and the higher the density is, the more accurate the mapping is. In addition, SLAF-seq has overcome the shortcomings of traditional markers, which are more time-consuming and labour-intensive. Therefore, SLAF-seq has been considered as a cost-effective technique to develop high stability and specificity molecular markers.

At present, tasting is a prevalent method for the comprehensive evaluation of sweet corn varieties by breeders. Although this method is direct and practical, its disadvantages include strong subjectivity and inaccurate phenotyping, making gene cloning difficult. Currently, with the rapid development of multiomics technologies (e.g., genomics, transcriptomics, metabolomics, proteomics, epigenomics, and lonomics), there are new opportunities to explore genes involved in the formation of pericarp thickness. Transcriptomics has been shown to be a powerful tool for the large-scale identification of genes related to 
specific traits in some crop species, including rice [17], maize [18], wheat [19], barley [20], and cotton [21]. To date, no RNA-seq study of pericarp thickness has been reported; thus, a more effective method to identify genes related to pericarp thickness is developed by integrating linkage analysis and transcriptome analysis. To identify the genes controlling the pericarp thickness of sweet corn and understand the genetic basis of the development of pericarp thickness in sweet corn. In this study, we constructed a $\mathrm{BC}_{4} \mathrm{~F}_{3}$ population using two sweet corn inbred lines with different pericarp thicknesses. Therefore, our aims are 1) to map pericarp thickness QTLs by linkage mapping and 2) to propose candidate genes for those QTLs based on complementary transcriptomic analyses. These results may provide molecular markers for sweet corn breeding with thinner pericarps and a theoretical basis for quality improvement and industrialization of sweet corn.

\section{Results}

\section{Phenotypic analysis}

The phenotypic data of the pericarp thickness of the $148 \mathrm{BC}_{4} \mathrm{~F}_{3}$ population and their two parents were collected in autumn of 2014, 2015 and 2016. As shown in Table 1, the pericarp thickness showed significant differences between the two parental lines across the 3 years. Compared with the 'M08' inbred line, the paternal parent 'M03' inbred line exhibited a lower pericarp thickness. The pericarp thickness of the $\mathrm{BC}_{4} \mathrm{~F}_{3}$ population ranged from 30.63 to $104.21 \mu \mathrm{m}$ and displayed a continuous distribution. The skewness and kurtosis of the trait ranged from $0.78-1.23$ and $1.17-2.29$, respectively, and the broad sense heritability $\left(h^{2}\right)$ of 3 years and cross years ranged from 0.66 to 0.73 (Table 1). The distributions of pericarp thickness for the $\mathrm{BC}_{4} \mathrm{~F}_{3}$ population were determined for samples over the 3 years and suggested that the segregation of this trait approximates the normal distribution and indicated that the pericarp thickness is a typical quantitative trait that is controlled by polygenes. A continuous distribution and considerable transgressive segregation were shown in the $\mathrm{BC}_{4} \mathrm{~F}_{3}$ population, suggesting that both parents contributed alleles towards pericarp thickness.
Positive correlations were observed among the traits evaluated over the 3 years (Additional file 1: Table S1). Correlation coefficients of pericarp thickness from the 3 years were found to be significant $(P<0.01)$ and were correlated with each other in a range from $r=0.756$ to 0.915. This result indicates that the pericarp thickness was stable among different environments.

\section{Identification of major pericarp thickness QTLs in the $\mathrm{BC}_{4} \mathrm{~F}_{3}$ population}

DNA of the $148 \mathrm{BC}_{4} \mathrm{~F}_{3}$ population was sent to a biomarker company for SLAF sequencing. A total of 163,961 SLAF tags were obtained, in which the coverage depth of the two parents was $42.15 x$, and the average sequencing depth of the offspring was $5.47 \times$. To improve the quality of the genetic map, SLAFs were filtered according to Zhu's method: 1) parents sequence depth $<10 \times$; 2) complete degree $<30 \%$; 3 ) partial separation significantly $(P<0.01) ; 4)$ heterozygous in two parents [16].

A total of 3876 SNPs were obtained after filtering the original SLAF tags. Based on the locations of SLAF markers on chromosomes, they were assigned into ten linkage groups (LGs) according to the maize inbred line B73 reference genome (version 3) (ftp://ftp.ensemblgenomes.org/ pub/plants/release-24/fasta/zea_mays/dna/). The linear arrangement of markers in the linkage group was obtained using JoinMap4.1 software, and the genetic distance between adjacent markers was estimated. Finally, a genetic map with a total map distance of $2413.25 \mathrm{cM}$ was constructed. The number of markers on the map was 3876 , and the average map distance between markers was 0.62 cM (Additional file 2: Fig. S1; Additional file 3: Table S2).

Based on the constructed genetic linkage map, the phenotype of the pericarp thickness of the $\mathrm{BC}_{4} \mathrm{~F}_{3}$ population collected from 2014, 2015, 2016 and the average of those 3 years were analysed by QTL mapping. A total of 14 QTLs for pericarp thickness were mapped in the $\mathrm{BC}_{4} \mathrm{~F}_{3}$ populations across 3 years. The QTLs were distributed on maize chromosomes 1, 5, 6, 7, and 10. Seven QTLs associated with pericarp thickness were found on chromosomes 1 and 10, and the phenotypic variance explained by a single QTL ranged from 3.36 to $7.78 \%$ in 2014. Three QTLs controlling pericarp thickness were identified on chromosomes

Table 1 Descriptive statistics of pericarp thickness for the parental lines and $\mathrm{BC}_{4} \mathrm{~F}_{3}$ populations

\begin{tabular}{|c|c|c|c|c|c|c|c|c|}
\hline \multirow[t]{2}{*}{ Trait } & \multicolumn{2}{|c|}{ Parents (Mean \pm SD) } & \multicolumn{3}{|c|}{$\mathrm{BC}_{4} \mathrm{~F}_{3}$ Population } & \multirow[t]{2}{*}{ Skewness } & \multirow[t]{2}{*}{ Kurtosis } & \multirow[t]{2}{*}{$h^{2}$} \\
\hline & $\mathrm{MO3}^{\mathrm{a}}$ & $M 08^{a}$ & Minimum $^{a}$ & Maximum $^{a}$ & Mean $\pm S D^{a}$ & & & \\
\hline 2014 FS & $57.86 \pm 7.28$ & $95.07 \pm 6.36$ & 46.17 & 104.21 & $70.80 \pm 10.17$ & 0.78 & 1.17 & 0.68 \\
\hline 2015 FS & $52.48 \pm 10.22$ & $97.81 \pm 13.36$ & 45.28 & 104.20 & $66.68 \pm 10.57$ & 0.91 & 2.29 & 0.66 \\
\hline 2016 FS & $47.29 \pm 11.23$ & $80.55 \pm 13.28$ & 30.63 & 94.66 & $50.04 \pm 12.31$ & 1.23 & 2.09 & 0.73 \\
\hline Average years & $52.54 \pm 9.58$ & $91.14 \pm 11.01$ & 44.19 & 99.26 & $67.41 \pm 10.81$ & 1.09 & 1.47 & 0.67 \\
\hline
\end{tabular}

${ }^{\mathrm{a}} \mu \mathrm{m}$

$h^{2}$ refer to broad sense heritability

A linear mixed model with random genotype three year interaction was used for analysis across three years 
5 , 6 , and 10 , accounting for $26.32 \%$ of phenotypic variance in 2015. Two QTLs ( $q P T 7$ and $q P T 10-5$ ) were responsible for pericarp thickness located on chromosomes 7 and 10, respectively, explaining a total of $21.76 \%$ of phenotypic variance in 2016. Two QTLs were detected based on the average pericarp thickness of 3 years, which explained 3.22 and $35.38 \%$ of phenotypic variance. A stable qPT10-5, which was located in a 901.2-kb (chr10: 145,172,996-145,532,401) region, was identified in all 3 years and averaged across 3 years and could explain 7.78 to $35.38 \%$ of phenotypic variations among different years. This finding indicated that qPT10-5 was the stable major locus interval controlling sweet corn pericarp thickness (Fig. 1; Table 2). According to the reference genome annotation information, forty-two genes within the region of qpt10-5 were found.

\section{Transcriptome profiling}

To identify the differentially expressed genes (DEGs) in qPT10-5, the pericarp 19 days after pollination (DAP) for two parents was used for transcriptome sequencing. Scanning electron microscopy (SEM) analysis of the pericarps of the M03 and M08 lines (each sample with five replications) at the 19th DAP was also performed, and the average pericarp thicknesses of M03 and M08 were 111.27 $\pm 9.19 \mu \mathrm{m}$ and $176.90 \pm 13.86 \mu \mathrm{m}$, respectively (Fig. 2). Approximately
$43.98 \mathrm{~Gb}$ of total nucleotide data were obtained from the M03 and M08 lines by RNA sequencing. Three independent biological replicates were used in this experiment. We obtained 49,153,314-54,655,798 reads for the inbred line M03, and 67.77 to $69.94 \%$ were mapped to the B73 reference genome (version 3). For the inbred line M08, we obtained 51,359,308-54,932,698 reads, and 70.06 to $70.46 \%$ were mapped to the B73 reference genome (Additional file 4: Table S3). A significantly high correlation (Additional file 5: Table S4) was observed between the two biological replications, revealing steady expression profiling between the replicated samples. In this study, a total of 4381 DEGs were identified between the M03 and M08 lines (|fold change $\mid \geq$ 2 and FDR $<0.01$ ). Among these genes, 2318 were upregulated and 2063 were downregulated in the M03 line.

Gene Ontology-based enrichment analysis was carried out using a threshold value $(\mathrm{FDR}<0.01)$ to evaluate the major biological functions of the DEGs (Fig. 3). These genes were further classified into three main categories, including biological processes (BP), cellular components (CC) and molecular functions (MF). The BP category contained half of the GO annotations $(18,418 ; 50.96 \%)$ followed by the CC category $(12,965 ; 35.87 \%)$ and the MF category $(4757 ; 13.17 \%)$. Under the biological processes category, cysteine biosynthetic process, response
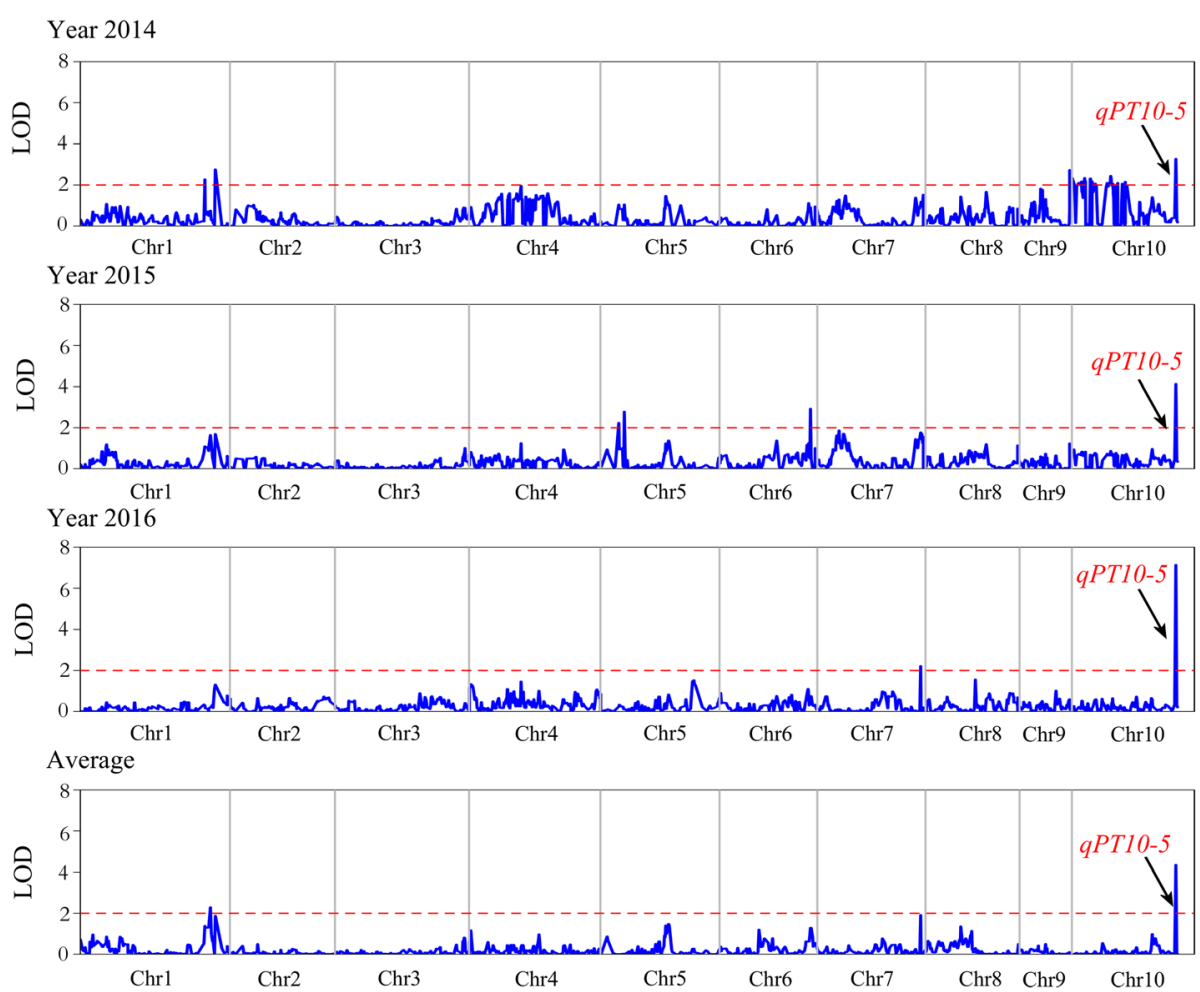

Fig. 1 Single-trait multiple-interval mapping of the QTL for pericarp thickness on ten chromosomes across three years in the $B C_{4} F_{3}$ population. Curves in the plot indicate the genetic coordinates along chromosomes within a chromosome ( $x$-axis) and LOD score (y-axis) of the detected QTL. The mapping curve of the QTL that controls pericarp thickness is located on chromosome 10, and a common qPT10-5 was detected across three years. The dashed line indicates the significance threshold $(L O D=2.0)$ 
Table 2 QTL detected for pericarp thickness in the $\mathrm{BC}_{4} \mathrm{~F}_{3}$ population

\begin{tabular}{|c|c|c|c|c|c|c|c|}
\hline Trait & QTL name & $\mathrm{Chr}^{\mathrm{a}}$ & $\mathrm{Cl}(\mathrm{bp})^{\mathrm{b}}$ & QTL region $(\mathrm{cM})$ & $\operatorname{LOD}^{c}$ & $\mathrm{ADD}^{\mathrm{d}}$ & $R^{2}(\%)^{e}$ \\
\hline \multirow[t]{7}{*}{ 2014FS } & qPT1-1 & 1 & $289,632,655-291,951,018$ & $292.01-296.98$ & 2.76 & 12.37 & 6.76 \\
\hline & aPT1-2 & 1 & $294,334,812-295,560,828$ & 273.97-274.01 & 2.27 & 3.02 & 3.36 \\
\hline & aPT10-1 & 10 & $73,141,877-74,489,414$ & $0-5.39$ & 2.72 & 13.02 & 6.72 \\
\hline & qPT10-2 & 10 & $51,051,991-53,058,997$ & $34.48-35.56$ & 2.22 & 12.97 & 6.64 \\
\hline & qPT10-3 & 10 & $49,740,097-50,781,644$ & $89.26-89.32$ & 2.16 & 12.96 & 6.69 \\
\hline & qPT10-4 & 10 & $31,389,610-33,625,026$ & $105.62-105.73$ & 2.1 & 12.95 & 6.65 \\
\hline & aPT10-5 & 10 & $144,631,242-145,532,401$ & $233.54-233.57$ & 3.27 & 14.00 & 7.78 \\
\hline \multirow[t]{3}{*}{ 2015FS } & QPT5 & 5 & $14,148,610-18,559,963$ & $39.86-42.01$ & 2.26 & 14.66 & 7.62 \\
\hline & qPT6 & 6 & $117,425,264$ & 204.24 & 2.92 & 16.69 & 9.88 \\
\hline & qPT10-5 & 10 & $144,631,242-145,532,401$ & $233.54-233.57$ & 4.12 & 15.74 & 8.82 \\
\hline \multirow[t]{2}{*}{ 2016FS } & qPT7 & 7 & $67,564,733-69,008,076$ & 231.99-232.04 & 2.21 & 14.62 & 7.92 \\
\hline & qPT10-5 & 10 & $144,631,242-145,532,401$ & $233.54-233.57$ & 7.13 & 22.61 & 13.84 \\
\hline \multirow[t]{2}{*}{ Average FS } & qPT1-3 & 1 & $295,869,898$ & 286.16 & 2.29 & 2.42 & 3.22 \\
\hline & qPT10-5 & 10 & $144,631,242-145,532,401$ & $233.54-233.57$ & 4.34 & 30.40 & 35.38 \\
\hline
\end{tabular}

\section{${ }^{\mathrm{a}}$ Chr chromosome}

${ }^{\mathrm{b}}$ The physical position corresponding to the $95 \%$ confidence interval for the detected QTL

'LOD the logarithm of odds score

${ }^{d}$ Positive and negative values indicated additive effects by the alleles of $\mathrm{M} 03$ and M08, respectively

${ }^{e} \mathrm{R}^{2}$ the phenotypic variance explained by an individual QTL

to salt stress, response to cadmium ion, responses to stimulus, Golgi organization, response to oxygencontaining compound, response to water deprivation, response to hypoxia, response to abscisic acid, and oxylipin biosynthetic process were prominently represented. Within the cellular components category, nucleus, cisGolgi network membrane, Golgi apparatus, endoplasmic reticulum, cell periphery, anchored component of plasma membrane, cytoplasmic membrane-bounded vesicle, Smc5-Smc6 complex, cytosol, and cell wall represented most of the subcategories. For the molecular functions category, many genes were classified into the protein binding, nutrient reservoir activity, carbohydrate derivative transporter activity, geranyltrans transferase activity, protein homodimerization activity, prunasin beta-glucosidase activity, 4-methylumbelliferyl-beta-Dglucopyranoside beta-glucosidase activity, esculin betaglucosidase activity, and cellobiose glucosidase activity subcategories.

KEGG enrichment analysis showed $(\mathrm{FDR}<0.01)$ that these genes were mainly located in plant hormone signal transduction, glycolysis/gluconeogenesis, pyruvate metabolism, valine, leucine and isoleucine degradation and fatty acid degradation pathways (Fig. 4).

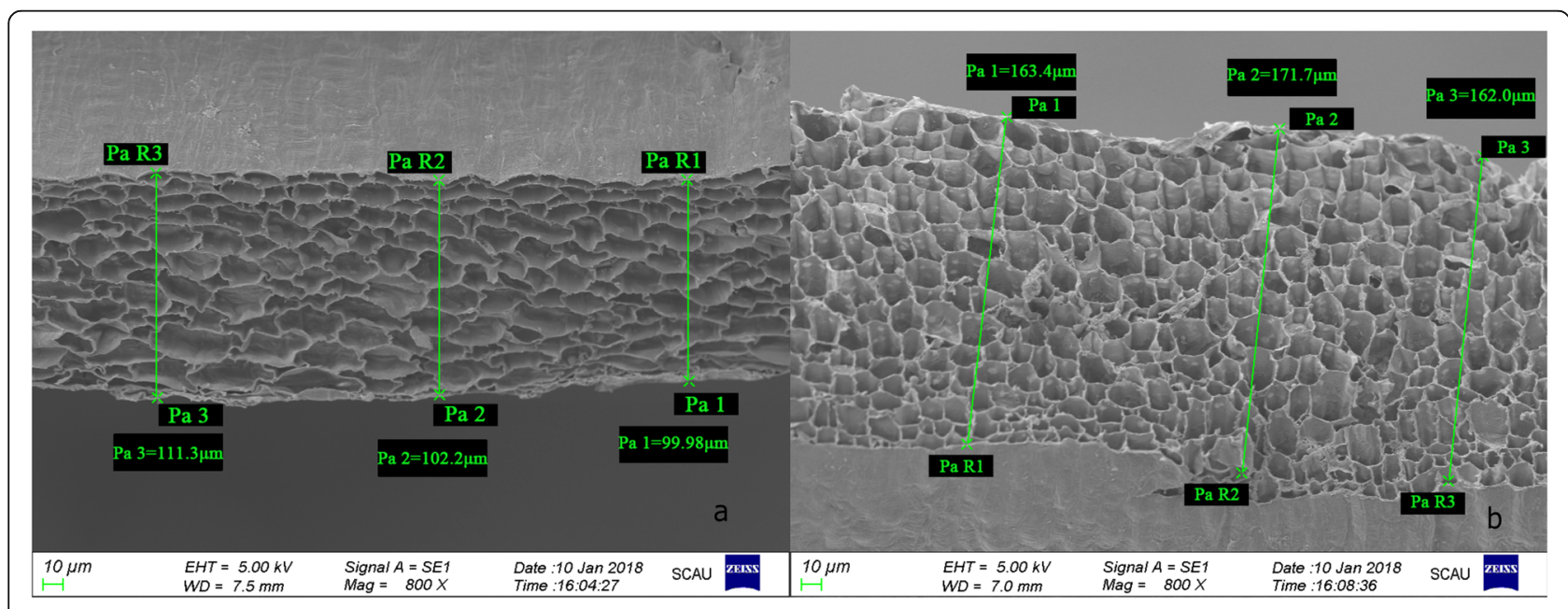

Fig. 2 Scanning electron microscopy of pericarp thickness of M03 and M08. a, b Pericarp thickness of M03 and M08 at 19 DAP, respectively 


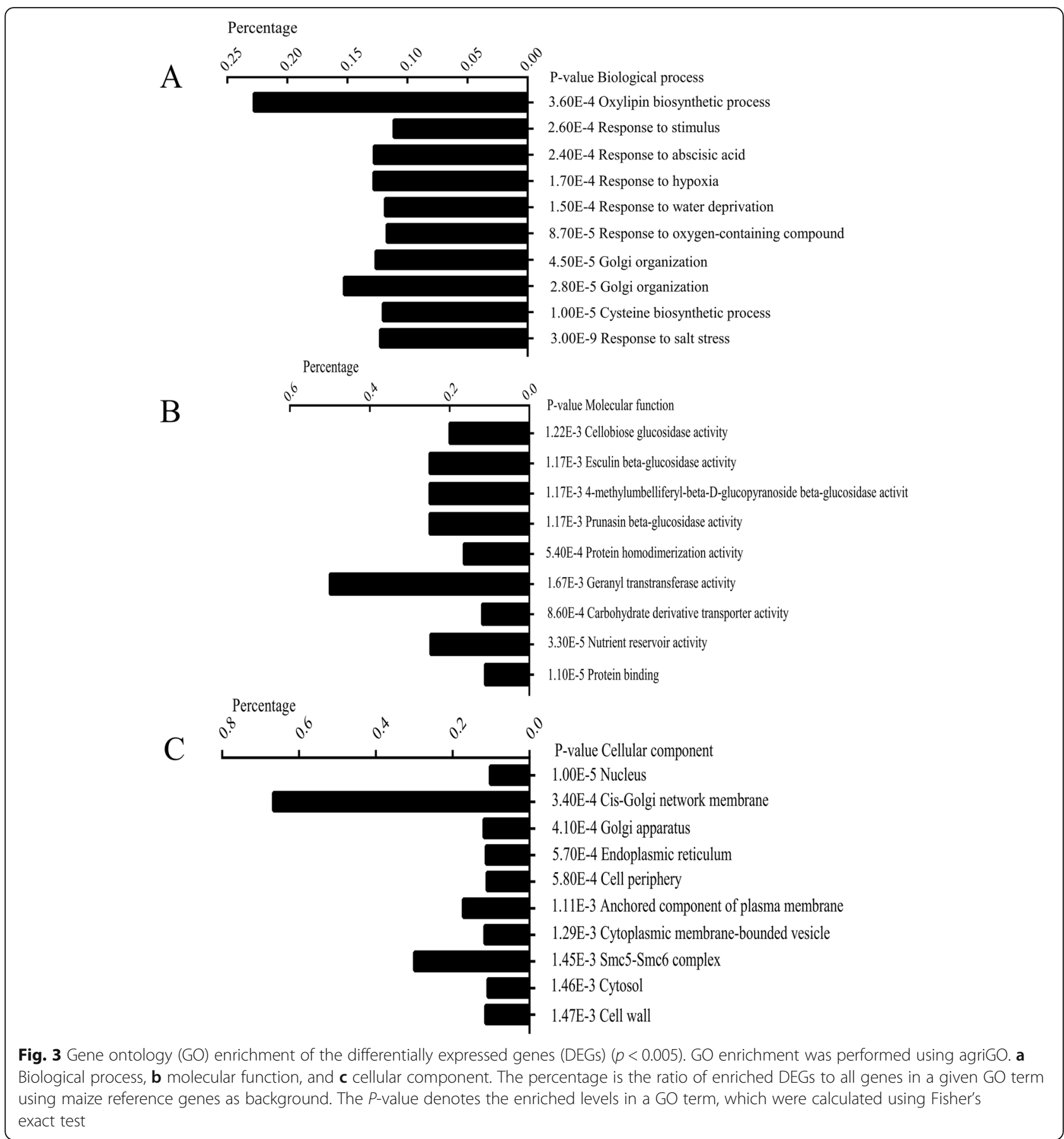

\section{Candidate gene prediction in qpt10-5 based on transcriptome analysis}

Forty-two candidate genes in the qpt10-5 region were compared with the DEGs identified by transcriptome sequencing. Among the 42 genes, 18 were found in the transcriptome sequencing (Additional file 6: Table S5). Only five genes, GRMZM6G287947, AC234202.1_FG004, GRM ZM2G143402, GRMZM2G143352 and GRMZM2G14 3389 , were differentially expressed between the M03 and M08 lines (Table 3). Gene annotation indicated that
GRMZM2G143352, GRMZM2G143402, and GRMZM2 G143389 may be candidate genes that control pericarp thickness. Of these genes, GRMZM2G143352 and AC234202.1_FG004 were upregulated in the M08 line, whereas GRMZM2G143389, GRMZM6G287947, and GRMZM2G143402 were downregulated in the M08 line. To confirm the results obtained from transcriptome sequencing, these genes were selected for qRT-PCR validation. As expected, the expression patterns of the 5 selected genes obtained from qRT-PCR were similar to 


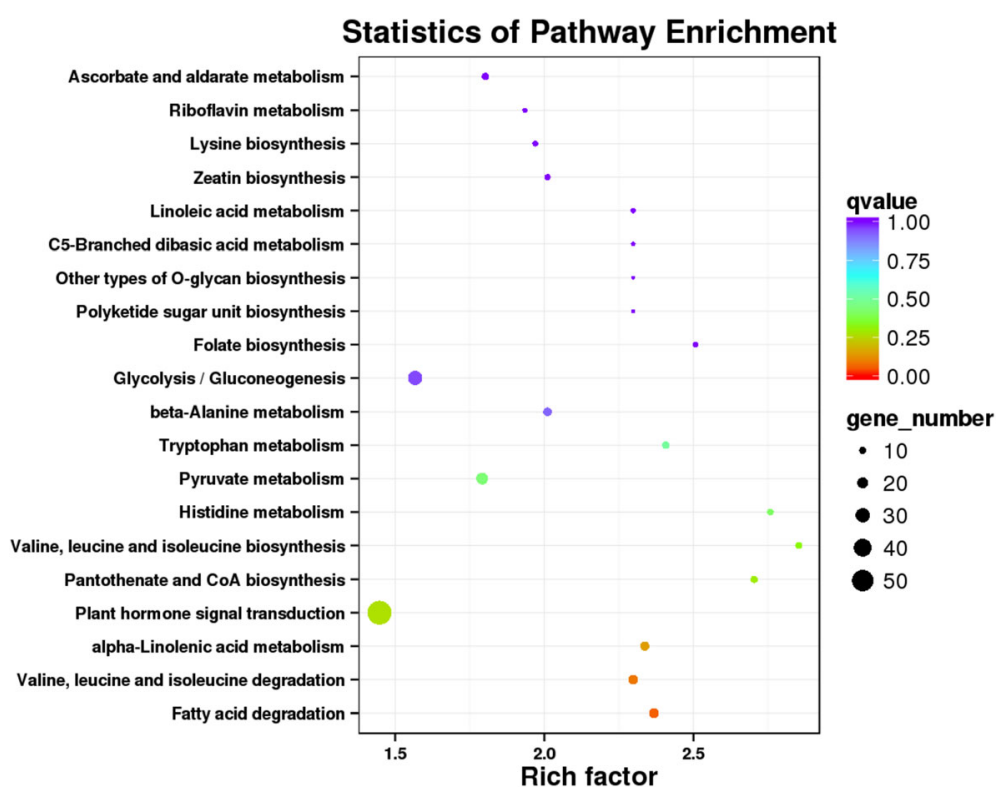

Fig. 4 Enriched KEGG pathways of target genes for differentially expressed genes. The most enriched pathway is the plant hormone signal transduction pathway

those obtained by transcriptome sequencing (Fig. 5). Furthermore, the full-length versions of those three genes in the M03 and M08 lines were sequenced. GRMZM2G143352 in the M08 line was missing a CGCG and ACCTCG sequence in front of the initiation codon and coding sequence compared with the sequence from the M03 line. Although the coding sequence was the same, GRMZM2G143402 in the M08 line had a 362-bp sequence insertion in the promoter, which contained a PIF-Harbinger transposon (Fig. 6). GRMZM2G143389 had a copy of CCGCTCA in the promoter of the M08 line compared with M03 (Additional file 7: Fig. S2). Variation in these three genes may lead to differences in pericarp thickness between the two parents. These results may facilitate the fine mapping of the qPT10-5 locus, and further experiments are needed to identify functional genes and identify causes of the differences in pericarp thickness.

\section{Discussion}

\section{Characteristics of the SLAF-seq method}

Compared to other sequencing techniques combined with restriction enzyme digestion (such as genotyping-bysequencing and restriction-site-associated DNA sequencing), SLAF-seq was measured by sequencing the specific length amplified fragment, which has better repeatability. Exploring numerous stable and reliable molecular markers is the key step for high-density genetic map construction. Once the genome is digested by restriction endonuclease(s), choosing fragments correctly for sequencing would be a better representation of the genome. In this study, the total number of SLAF markers was more than 520,000, and the number of polymorphic markers was 31,582. This result is difficult to achieve with traditional molecular markers. Furthermore, all the markers covered the whole genome, which ensured the accuracy of the final mapping. The labels with poor representativeness and inadequate completeness and

Table 3 Different expressed genes within the interval of QPT10-5 in sampled pericarp for M03 and M08

\begin{tabular}{lllllll}
\hline Gene ID & M03 $^{\mathrm{a}}$ & M08 $^{\mathrm{a}}$ & FDR & Log $_{2} \mathrm{FC}^{\mathrm{b}}$ & Regulated $^{\mathrm{c}}$ & Gene Annoation $^{\text {GRMZM2G143352 }}$ \\
\hline 0.29 & 4.4 & $8.33 \mathrm{E}-05$ & 4.10 & up & Auxin-responsive protein IAA33 \\
GRMZM2G143389 & 17.21 & 2.53 & $4.88 E-07$ & -2.83 & DOWN & Protein FATTY ACID EXPORT 2 chloroplastic \\
GRMZM2G143402 & 132.88 & 36.08 & $2.19 \mathrm{E}-09$ & -1.89 & DOWN & ZIM-transcription factor 34 \\
GRMZM6G287947 & 202.77 & 0.68 & $8.76 E-14$ & -8.73 & DOWN & None \\
AC234202.1_FG004 & 0 & 2.51 & $1.64 E-12$ & Inf & up & Hypothetical protein ZEAMMB73_137218 \\
\hline
\end{tabular}

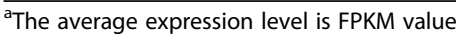

${ }^{b}$ Fc FPKM change between M03 and M08

${ }^{\mathrm{C}}$ NS Non-significant, UP Upregulated expression, DOWN Downregulated expression 


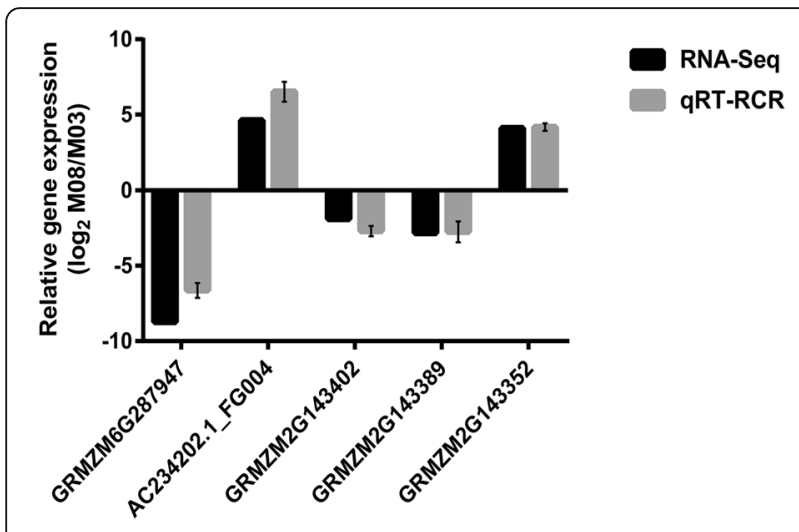

Fig. 5 Verification of relative expression levels of DEGs by qRT-PCR. Error bars indicate standard deviation from 3 biological and technical replicates of qRT-PCR. Expression patterns of 10 DEGs by qRT-PCR (black bar) and RNA-Seq (grey bar)

the bias separation labels could be filtered out. All of these beneficial properties simplified the genome sequencing, which proves the superiority of this technique in genetic mapping. On the other hand, due to the large number of markers, complex populations and other factors, the sequencing results also increased the difficulty and challenge of the mapping analysis. Although the construction of the linkage map and its application in common corn have been widely applied [22, 23], few studies have been published about genetic mapping of the whole genome in sweet corn. In this study, we provide a reference for follow-up genetic research on sweet corn.

\section{Pericarp thickness QTL mapping results compared with other studies}

With the rapid development of molecular biotechnology and bioinformatics, plant gene mapping has made considerable progress. Previous studies have shown that a total of $190 \mathrm{BC}_{1} \mathrm{~F}_{2}$ families crossed by two cultivars with different pericarp thicknesses were used as the mapping population. Eight QTLs were found to be linked to pericarp thickness and mapped to chromosomes 1, 2, 3, 5, 6 and 8 [12]. In addition, 33 QTLs were detected by 264 $\mathrm{F}_{2: 3}$ families crossed by the Korean waxy maize inbred lines $\mathrm{BH} 20$ and $\mathrm{BH} 30$, and these loci were located on chromosomes $1,2,3,4,8,9$, and 10 [11]. In this study, based on the previous research conclusions, pericarp thickness of the upper abgerminal part was regarded as a representative to determine the sweet corn pericarp thickness. Finally, a major QTL locus was found on chromosome 10 and could explain 7.78 to $13.84 \%$ of phenotypic variations among different years. Phenotypic variance explained by a QTL was estimated based on the same data that were used to detect the QTL, which could cause the $R^{2}$ values to be biased upwards [24]. Among the chromosomes, 10 chromosomes had the most frequently linked markers. This result is different from that of $\mathrm{Yu}$ et al. but is similar to that of Choe and Rocheford [11, 12]. It was found that the loci mapped on the same chromosome were generally adjacent to their predecessors. Therefore, the chromosome may have the same major gene loci as previous generations.

\section{Candidate gene analysis}

GRMZM2G143352 is an AUX/IAA transcription factor, GRMZM2G143402 is a ZIM transcription factor, and GRMZM2G143389 is the chloroplastic FATTY ACID EXPORT 2 protein. Both GRMZM2G143352 and GRMZ $M 2 G 143402$ were related to plant hormone signal transduction, and GRMZM2G143389 was related to fatty acid degradation pathways, which is consistent with the results of the KEGG enrichment analysis (Fig. 4). Furthermore, the full-length gene sequences of these three genes are different between the M03 and M08 lines.

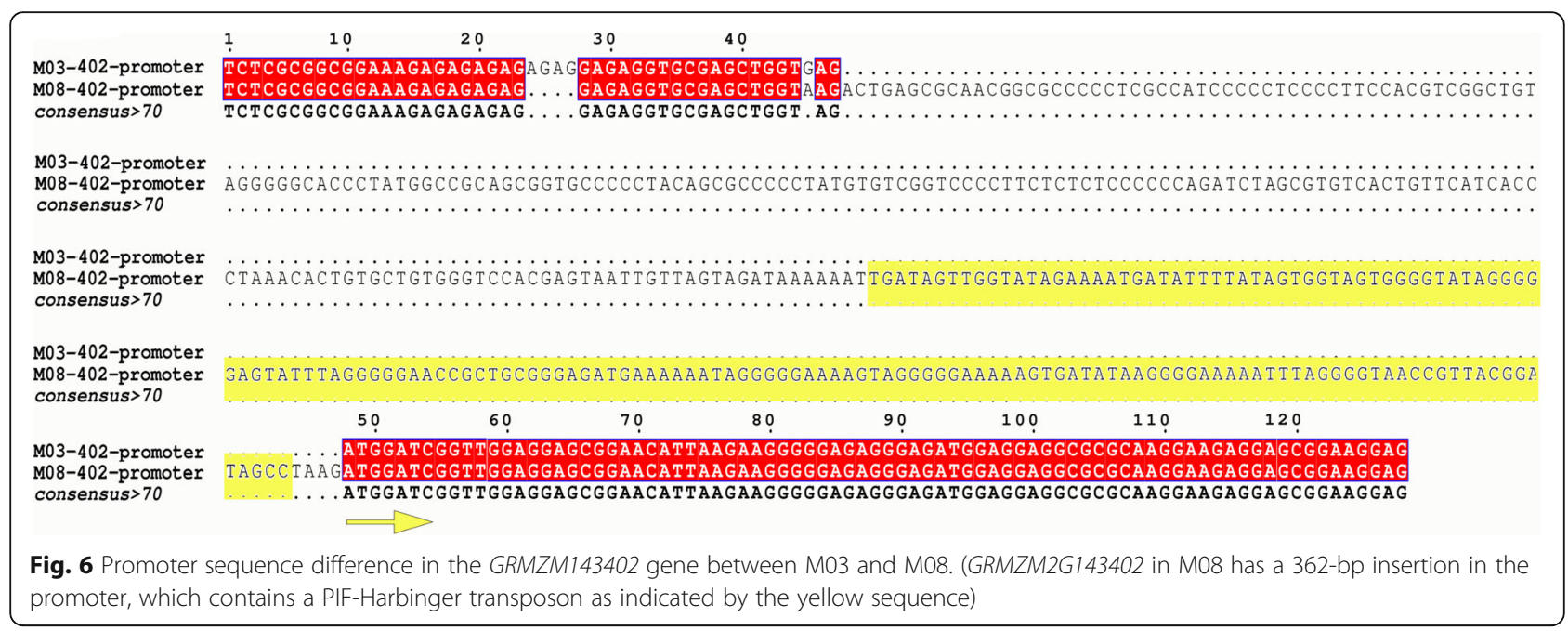


Variation in these three genes may lead to differences in pericarp thickness between the two parents.

GRMZM2G143352 is an AUX/IAA transcription factor that mediates many aspects of plant responses to auxin [25]. The functions of most Aux/IAAs have been defined mainly by gain-of-function mutant alleles in Arabidopsis thaliana [26]. Two different classes of transcription factors are involved in the regulation of auxin signalling, AUX/IAAs and AUXIN RESPONSE FACTORS (ARFs). ARFs bind directly to auxin response elements in the promoter region of auxin-responsive genes via their conserved DNA-binding domain (DBD). When auxin is low, AUX/IAA proteins bind to ARFs, preventing the expression of auxin-responsive genes. A high level of auxin promotes ubiquitination and degradation of AUX/IAAs though SCF ${ }^{\mathrm{TIR} 1 / \mathrm{AFB}}$ and the $26 \mathrm{~s}$ proteasome, leading to the activation of auxin-response genes by ARFs [27, 28]. These response genes may be related to pericarp development.

We know that auxin treatment can promote the expression of AUX/IAA genes [29, 30]. We found that the expression level of GRMZM2G143352 in the M08 line was higher than that in the M03 line, indicating that the auxin level of the M08 line could be higher than that in the M03 line. Auxin is an important cell cycle regulator [31]. The level of auxin affects cell growth, lowers concentrations of indole-3-acetic acid (IAA) and can promote the elongation of tobacco BY-2 cells but has no effect on cell division. However, cell division was accelerated, but cell elongation was inhibited at relatively high IAA concentrations [32, 33]. These findings are consistent with our results (Fig. 3).

GRMZM2G143402 is a ZIM transcription factor that is a repressor of JA (jasmonate) in maize [34]. The JA signal regulates plant growth, development and defence responses. JAZ (jasmonate ZIM domain) transcription repressors bind directly to JA-responsive genes. Without the JA ((3R,7S)-jasmonoyl-L-isoleucine) signal, JAZ regulates the JA-mediated response by inhibiting the transcriptional activity of JA signalling-responsive transcription factors $[35,36]$. When the JA signal appeared, the JA receptor specifically combined with JAZ, resulting in JAZ ubiquitination and degradation by proteasomes, relieving JAZ's inhibition of JA transcriptional regulation and causing physiological changes [37]. Through regulating the development of the stomata, JA can improve the ability of plants to cope with various external stresses [38]. Some components of the JA signalling pathway can be independently involved in plant stomatal development regulation [39]. The size of the stoma and the open state may be one of the reasons for the difference in pericarp thickness.

According to the gene annotation, GRMZM2G143389 is the fatty acid export 2 (FAX2) chloroplastic protein. Fatty acid synthesis in plants occurs in plastids; therefore, it needs to export for acyl editing and lipid assembly in the cytoplasm and endoplasmic reticulum. However, plastid fatty acids' transport mechanism has not been elucidated. The function of fatty acid output 1 ( $f a x 1)$ is essential for biomass and male reproductive capacity [40], while $F A X 2$ function is unknown. Hence, it would be highly interesting if we could prove the association of this gene with sweet corn pericarp thickness. Therefore, additional evidence is required to demonstrate the potential role of these genes in the pericarp thickness of sweet corn.

\section{Conclusions}

In our study, we created a $\mathrm{BC}_{4} \mathrm{~F}_{3}$ population and constructed a high-density genetic linkage map that had an average genetic distance of $0.62 \mathrm{cM}$ between adjacent markers by the SLAF approach. Based on this highdensity genome map, a total of 14 QTLs for pericarp thickness were detected, and one stable QTL (qPT10-5) was detected across multiple years, which explained 7.78$35.38 \%$ of the phenotypic variation located on chromosome 10. Moreover, five genes of the target region of qPT10-5 were differentially expressed between the two parents, as revealed by transcriptome analysis. According to the gene annotation information, three genes might be considered candidates for pericarp thickness. This study identified a major QTL and candidate genes that could accelerate breeding for thin pericarp thickness varieties of sweet corn, and these results established the basis for map-based cloning and further functional research.

\section{Methods}

\section{Plant material}

Two sweet corn inbred lines, M03 and M08, were selected as parents to develop a $\mathrm{BC}_{4} \mathrm{~F}_{3}$ mapping population. The two lines show different pericarp thicknesses; as the recurrent parent, M03 is a thinner pericarp thickness; as the donor parent, M08 is a thicker pericarp thickness line. All plant materials used in this study were generously provided by Prof. Xiaoqin Li (College of Agriculture, South China Agricultural University, Guangzhou, China). The mapping population consisted of 148 lines, and evaluation of background recovery rates showed only 8 lines whose genetic background recovery rates were less than $90 \%$; the recovery rates of the other lines were higher than $90 \%$, the highest rate was $99.99 \%$, and the average background recovery rate was $95.91 \%$. These results showed that the genetic background of the $\mathrm{BC}_{4} \mathrm{~F}_{3}$ population was largely the same as that of the recipient parents.

Two parents and 148 lines were grown in the Zengcheng Experimental Base of South China Agricultural University (at approximately $113^{\circ}$ east longitude and approximately $23^{\circ}$ north latitude) in autumn of 2014, 2015, and 2016. Two parents planted in 2017 were used for transcriptome sequencing. A randomized complete-block 
design was used. Each line or parent was grown in 10 plants with 2 replicates. The length of the rows was $3 \mathrm{~m}$, and the row spacing was $70 \mathrm{~cm}$. The plant spacing was 25 $\mathrm{cm}$, and the density was $57,000 \mathrm{hm}^{2}$. Crop management and disease and insect pest control were performed as locally recommended.

Three self-pollinated ears of the two parents were sampled and placed immediately on ice 19 days after pollination in the field in 2017. Ten kernels were collected from the middle of each ear. The upper abgerminal part of the pericarp was stripped on ice and stored in liquid nitrogen for analysis by scanning electron microscopy.

\section{Phenotypic data collection Frozen section method}

Ten whole kernels from each ear were placed on ice. Each kernel was cut off approximately $3 \mathrm{~mm}$ at the top with bald tweezers and then transferred into liquid nitrogen for $3 \mathrm{~s}$. Frozen samples were used for the preparation of tissue slices. We took cross-sections from the dorsal embryo with a thickness of approximately $100 \mu \mathrm{m}$. The process was completed before thawing to ensure that the pericarp did not fall off. The slices were then quickly placed on the slide. After thawing, $\mathrm{KI}-\mathrm{I}_{2}$ reagent was added for $3 \mathrm{~s}$, and the dye was then washed off with water after covering the coverslip. The pericarp thickness of each kernel was measured by micrometer. The average value of pericarp thickness from three ears of each genotype was regarded as the observed value of the pericarp thickness and used for subsequent analysis $[2,41]$.

\section{Phenotypic data analysis}

The phenotypic data were analyzed using SPSS version 19 (SPSS Inc., Chicago, IL, USA). These variance components of the genotype and environment in each year were estimated by using the linear mixed model: $\mathrm{y}_{\mathrm{il}}=\mu+r_{l}+f_{i}+\varepsilon_{i l}$, and these variance components of the genotype and environment in 3 years were estimated by using the following mixed model: $\mathrm{y}_{\mathrm{ijl}}=\mu+e_{j}+r_{l(j)}+f_{i}+(f e)_{i j}+\varepsilon_{i j l}$, where $\mu$ is the grand mean of pericarp thickness, $f_{i}$ is the genetic effect of the $i$ th line, $r_{l}$ is the effect of replications, $\varepsilon_{i l}$ is the residual error, $e_{j}$ is the environmental effect of the $j$ th environment, $(f e)_{i j}$ is the interaction effect between genetic and environmental effects, $r_{l(j)}$ is effect of replications within environments, and $\varepsilon_{i j l}$ is the residual error [42]. The PROC MIXED procedure in SAS software (Release 9.4; SAS Institute, Cary, NC) was used to get the variance components of all pericarp thickness traits.

The broad sense heritability $\left(h^{2}\right)$ in each year was estimated using the following formula: $h^{2}=\sigma_{g}^{2} /\left(\sigma_{g}^{2}+\sigma_{z}^{2} / r\right)$, and the $h_{b}^{2}$ in cross-years was estimated using the following formula: $h_{b}^{2}=\sigma_{g}^{2} /\left(\sigma_{g}^{2}+\sigma_{g e}^{2} / e+\sigma_{z}^{2} / r e\right)$, where $\sigma_{g}^{2}$ is the genetic variance, $\sigma_{z}^{2}$ is the residual error, and $\mathrm{r}$ is the number of replications, ${\sigma_{\mathrm{ge}}}^{2}$ is the interaction of genotype with environment, $e$ and $r$ represent the number of environments and replications in each environment [42].

\section{Library construction and high-throughput sequencing}

Genomic DNA was extracted from the two parents and the $\mathrm{BC}_{4} \mathrm{~F}_{3}$ population using a cetyl trimethylammonium bromide (CTAB) protocol [43]. Illumina $\mathrm{HiSeq}^{\mathrm{Tm}} 2500$ sequencing was used for DNA sequencing. The Hpy166II endonuclease was chosen to digest the genomic DNA after profiling the restriction endonuclease sites on the reference genome. Details of the SLAF-seq strategy and library construction were described previously [13].

\section{Grouping and genotyping of sequence data}

Specific length amplified fragment (SLAF) markers were identified and genotyped following procedures described by Sun et al. [13] and Zhang et al. [44]. The remaining reads were mapped to the reference genome using BWA software after filtering out the low-quality reads [45]. Sequences that had greater than $95 \%$ similarity were defined as the same SLAFs. All polymorphic SLAF markers that were consistent with parents and offspring were genotyped.

All SLAF markers were filtered four times, and the quality was assessed as described by Sun et al. [13]. In brief, markers with fewer than 3 SNPs and an average sequencing depth higher than 3 were treated as highquality SLAF markers. These markers were used to construct high-density genetic maps.

\section{Linkage map construction}

The imputation of missing genotypes was performed using the K-nearest neighbour algorithm based on the two parents and the $\mathrm{BC}_{4} \mathrm{~F}_{3}$ population [46]. A linkage map was constructed according to the procedure described by Zhang et al. [44]. In detail, the SLAF markers in the linkage groups were ordered using the High Map Strategy [47] and SMOOTH strategy [48]. Multipoint method of maximum likelihood was used to add these skewed markers into the linkage map [49]. The genetic distance between the adjacent markers was estimated by kosambi mapping function [50].

\section{QTL analysis}

QTL mapping was carried out by the composite interval mapping method in QTLNetwork v2.1 software for a pericarp thickness of three years and an average of three years [51]. 1000 permutations with the mapping step of $1.0 \mathrm{cM}$ was applied to calculate the LOD threshold. By default, a $10 \mathrm{cM}$ window with background marker set to 5 , and a genome-wide significance level of $P<0.05$ [52]. QTLs with the LOD score $>2.0$ were considered as suggestive QTLs [14]. Moreover, the mode of QTL action 
was determined according to the criteria proposed by Stuber et al. [53]. The QTL is named by q and abbreviated by traits. In addition, the chromosome number of the QTL and multiple QTLs on the same chromosome are designated by 1, 2, 3, and so on. The QTL names in this paper are expressed in italics; for example, qpt1-3 indicates the third QTL on chromosome 1 detected in the controlled pericarp thickness population.

\section{Transcriptome analysis}

Total RNA was isolated from the pericarps (19th DAP) using the Plant Total RNA Purification Kit (TR02-150, GeneMarkbio). The extracted RNA was run in a $1 \%$ agarose gel to assess the integrity of the RNA. Briefly, poly-A RNA-containing mRNA was enriched using poly-Toligo-attached magnetic beads and fragmented. Second-strand cDNA was synthesized using random hexamer primers and then purified, end-repaired, poly-A tailed, and adaptor-ligated. The cDNA libraries were sequenced with a read length of $100 \mathrm{bp}$ (paired-end) using the Illumina HiSeq 2000 System at Biomarker Technologies (Beijing, China). The experiment was performed with three biological replicates.

\section{Quantitative reverse-transcriptase PCR}

Quantitative reverse-transcriptase PCR (qRT-PCR) was carried out to validate the RNA-seq results. The total RNA from each sample was extracted and reverse transcribed into single-stranded cDNA using a FastQuant RT Kit (Takara) including gDNase according to the manufacturer's protocol. Gene expression was determined by qRT-PCR analysis using the CFX96 Real-Time System (Bio-Rad). All reactions were performed in $20-\mu \mathrm{L}$ volumes consisting of $1 \mu \mathrm{l} \mathrm{cDNA}, 0.3 \mu \mathrm{M}$ of each gene-specific primer and the SsoFast EvaGreen Supermix Kit (Bio-Rad). qRT-PCR was conducted with the following protocol: $94^{\circ} \mathrm{C}$ for $1 \mathrm{~min}$ followed by 40 cycles of $95^{\circ} \mathrm{C}$ for $10 \mathrm{~s}, 55^{\circ} \mathrm{C}$ for $10 \mathrm{~s}$, and $72{ }^{\circ} \mathrm{C}$ for $15 \mathrm{~s}$. The relative transcriptional levels were calculated using the $2^{-\triangle \Delta C T}$ method [54] with actin as a housekeeping gene. The specific primers were designed using NCBI primer BLAST (http://www.ncbi.nlm.nih.gov/tools/ primer-blast/). The primer sequences for each gene are listed in Additional file 8 (Table S6).

\section{Supplementary information}

Supplementary information accompanies this paper at https://doi.org/10. 1186/s12870-020-2295-8.

Additional file 1: Table S1. Correlation coefficients of pericarp thickness measured among the three years.

Additional file 2: Fig. S1. Genetic map of the 148 CSSLs. The black stripe shows the distribution of markers on the 10 chromosomes.

Additional file 3: Table S2. Basic information of the genetic map.
Additional file 4: Table S3. RNA-seq reads of sweet corn pericarp mapped to the maize B73 RefGen_V3 genome.

Additional file 5: Table S4. Pearson correlations for the RNAsequencing results among different samples.

Additional file 6: Table S5. Expressed genes within the interval of qPT10-5 in sampled pericarp for M03 and M08.

Additional file 7: Fig. S2. Promoter sequence difference in the GRMZM143389 gene between M03 and M08. (The green box is a copy of CCGCTCA, and the yellow box has an inserted CTCGAGCAG sequence).

Additional file 8: Table S6. Primers for quantitative real-time RT-PCR (qRT-PCR) validation.

\section{Abbreviations}

ARFs: Auxin response factors; CIM: Composite interval mapping method; DAP: Days after pollination; DEGs: Different expression genes; FAX1: Fatty acid export 1; FAX2: Fatty acid export 2; FS: Frozen section; IAA: Indole-3acetic acid; JAZ: Jasmonate ZIM domain; LOD: Base 10 log likelihood ratio; QTL: Quantitative trait loci; RFLP: Restriction fragment length polymorphism; SEM: Scanning electron microscope; SLAF: Specific length amplified fragment; SNP: Single nucleotide polymorphism; SSR: Simple sequence repeats

\section{Acknowledgements}

We thank Biomarker Technologies Corporation (Beijing, China) for their support in SLAF sequencing.

\section{Authors' contributions}

WX conducted the experiments, analyzed the data, and drafted the manuscript; WB, XF and GJ conducted the experiments; FF and $\mathrm{HJ}$ analyzed the data; WX, $X F, Z L$ and $Z W$ conducted the field trials; $L X, F F$, and $H J$ designed the experiments, analyzed the data and drafted the manuscript. All authors revised the manuscript. All authors have read and approved the manuscript.

\section{Funding}

This work was financially support by the National Key Research and Development Program of China(Grant No.2018YFD0100106), the Keypoint Research and Invention Program of Guangdong Province (2018B020202013) and training plan for doctoral innovative talents in South China Agricultural University (CX2019N056). Authors declare that none of the funding bodies have any role in the research design, the data collection and analysis, and the manuscript preparation.

\section{Availability of data and materials}

All SLAF-seq raw data from this study has been submitted to the NCBI Sequence Read Archive (SRA) database under BioProject accession number PRJNA574257 (https://www.ncbi.nlm.nih.gov/bioproject/PRJNA574257). The raw RNA-sequencing data were deposited in NCBI SRA database under BioProject accession number PRJNA605850 (https://www.ncbi.nlm.nih.gov/bioproject/ ?term=PRJNA605850). The maize reference genome B73 RefGen_V3 sequence and annotation files were downloaded from ftp://ftp.ensemblgenomes.org/ pub/plants/release-24/fasta/zea_mays/dna/ and ftp://ftp.ensemblgenomes.org/ pub/plants/release-24/gtf/zea_mays/.

Ethics approval and consent to participate

Not applicable.

\section{Consent for publication}

Not applicable.

\section{Competing interests}

The authors declare no competing interests.

\section{Author details}

${ }^{1}$ The State Key Laboratory for Conservation and Utilization of Subtropical Agro-bioresources, South China Agricultural University, Guangzhou 510642, Guangdong, People's Republic of China. ${ }^{2}$ The Key Laboratory of Plant Molecular Breeding of Guangdong Province, College of Agriculture, South China Agricultural University, Guangzhou 510642, Guangdong, People's Republic of China. 


\section{Received: 29 April 2019 Accepted: 19 February 2020}

Published online: 14 March 2020

\section{References}

1. Bailey D. The relation of the pericarp to tenderness in sweet corn. J Am Soc Hortic Sci. 1938;36:555-9.

2. Helm J, Zuber M. Inheritance of pericarp thickness in corn belt maize 1. Crop Sci. 1972;12(4):428-30.

3. Ho L, Kannenberg L, Hunter R. Cytology. Inheritance of pericarp thickness in short season maize inbreds. Can J Genet Cytol. 1975;17(4):621-9.

4. Ito GM, Brewbaker J. Genetic analysis of pericarp thickness in progenies of eight corn hybrids. J Am Soc Hortic Sci. 1991;116(6):1072-7.

5. Haddad ES. Morphological development of sweet corn pericarp in two inbred lines and their $F_{1}$ hybrid. Indiana Agr Exp Sta Bull. 1931;347.

6. Tracy W, Schmidt D. Effect of endosperm type on pericarp thickness in sweet corn inbreds 1. Crop Sci. 1987;27(4):692-4

7. Azanza F, Tadmor Y, Klein B, Rocheford T, Juvik J. Quantitative trait loci influencing chemical and sensory characteristics of eating quality in sweet corn. Genome. 1996;39(1):40-50.

8. Wang B, Brewbaker J. Quantitative trait loci affecting pericarp thickness of corn kernels [Zea mays L.]. Maydica. 2001;46:159-65.

9. Rocheford T. QTL analysis of pericarp thickness and ear inflorescence architecture in South Korean waxy corn germplasm. Paper presented at the ASA-CSSA-SSSA International Annual Meetings 2006 Indianapolis, IN, 12-16November 2006.

10. Choe E. Marker assisted selection and breeding for desirable thinner pericarp thickness and ear traits in fresh market waxy corn germplasm. Doctoral dissertation, University of Illinois at Urbana-Champaign. 2010.

11. Choe E, Rocheford TR. Genetic and QTL analysis of pericarp thickness and ear architecture traits of Korean waxy corn germplasm. Euphytica. 2012; 183(2):243-60

12. YU Y, Li G, Qi X, Li X. Mapping and epistatic interactions of QTLs for pericarp thickness in sweet corn. Acta Agrono Sinica. 2015;41:359-66.

13. Sun X, Liu D, Zhang X, Li W, Liu H, Hong W, Jiang C, Guan N, Ma C, Zeng H. SLAF-seq: an efficient method of large-scale de novo SNP discovery and genotyping using high-throughput sequencing. PLoS One. 2013;8(3):e58700.

14. Zhang Z, Shang H, Shi Y, Huang L, Li J, Ge Q, Gong J, Liu A, Chen T, Wang D. Construction of a high-density genetic map by specific locus amplified fragment sequencing (SLAF-seq) and its application to Quantitative Trait Loci (QTL) analysis for boll weight in upland cotton (Gossypium hirsutum.). BMC Plant Biol. 2016;16(1):79.

15. Guo Y, Shi G, Liu Z, Zhao Y, Yang X, Zhu J, Li K, Guo X. Using specific length amplified fragment sequencing to construct the high-density genetic map for Vitis (Vitis vinifera L.x Vitis amurensis Rupr.). Front Plant Sci. 2015. 6:393.

16. Zhu WY, Huang L, Chen L, Yang JT, Wu JN, Qu ML, Yao DQ, Guo CL, Lian $\mathrm{HL}$, He HL. A high-density genetic linkage map for cucumber (Cucumis sativus L.): based on specific length amplified fragment (SLAF) sequencing and QTL analysis of fruit traits in cucumber. Front Plant Sci. 2016;7:437.

17. Zhang X, Rerksiri W, Liu A, Zhou X, Xiong H, Xiang J, Chen X, Xiong X. Transcriptome profile reveals heat response mechanism at molecular and metabolic levels in rice flag leaf. Genes. 2013;530(2):185-92.

18. Frey FP, Urbany C, Hüttel B, Reinhardt R, Stich B. Genome-wide expression profiling and phenotypic evaluation of European maize inbreds at seedling stage in response to heat stress. BMC Genomics. 2015;16(1):123.

19. Qin D, Wu H, Peng H, Yao Y, Ni Z, Li Z, Zhou C, Sun Q. Heat stressresponsive transcriptome analysis in heat susceptible and tolerant wheat (Triticum aestivum L.) by using Wheat Genome Array. BMC Genomics. 2008; 9(1):432.

20. Mangelsen E, Kilian J, Harter K, Jansson C, Wanke D, Sundberg E. Transcriptome analysis of high-temperature stress in developing barley caryopses: early stress responses and effects on storage compound biosynthesis. Mol Plant. 2011:4(1):97-115.

21. Nigam D, Kavita P, Tripathi RK, Ranjan A, Goel R, Asif M, Shukla A, Singh G, Rana D, Sawant SV. Transcriptome dynamics during fibre development in contrasting genotypes of G ossypium hirsutum L. Plant Biotechnol J. 2014; 12(2):204-18.

22. Chen Q, Song J, Du WP, Xu LY, Jiang Y, Zhang J, Xiang XL, Yu GR. Identification and genetic mapping for rht-DM, a dominant dwarfing gene in mutant semi-dwarf maize using QTL-seq approach. Genes Genomics. 2018;40(10):1091-9.
23. Cui Z, Xia A, Zhang A, Luo J, Yang X, Zhang L, Ruan Y, He Y. Linkage mapping combined with association analysis reveals QTL and candidate genes for three husk traits in maize. Theor Appl Genet. 2018;131(10):213144.

24. Utz HF, Melchinger AE, Schön CC. Bias and sampling error of the estimated proportion of genotypic variance explained by quantitative trait loci determined from experimental data in maize using cross validation and validation with independent samples. Genetics. 2000; 154(4):1839-49.

25. Sekhon RS, Lin H, Childs KL, Hansey CN, Buell CR, Leon N, Kaeppler SM. Genome-wide atlas of transcription during maize development. Plant J. 2011;66(4):553-63.

26. Wang H, Jones B, Li Z, Frasse $\mathrm{P}$, Delalande $\mathrm{C}$, Regad F, Chaabouni S, Latche A, Pech JC, Bouzayen M. The tomato aux/IAA transcription factor IAA9 is involved in fruit development and leaf morphogenesis. Plant Cell. 2005; 17(10):2676-92.

27. Ulmasov T, Hagen G, Guilfoyle T. ARF1, a transcription factor that binds to auxin response elements. Science. 1997;276(5320):1865-8.

28. Weijers D, Friml J. SnapShot: auxin signaling and transport. Cell. 2009;136(6): 1172

29. Abel S, Theologis A. Early genes and auxin action. Plant Physiol 1996, 111(1): 9-17.

30. Hagen G, Guilfoyle T. Auxin-responsive gene expression: genes, promoters and regulatory factors. Plant Mol Biol. 2002;49(34):373-85.

31. Francis D, Sorrell DA. The interface between the cell cycle and plant growth regulators: a mini review. Plant Growth Regul. 2001;33(1):1-12.

32. Chen J. Dual auxin signaling pathways control cell elongation and division. Plant Growth Regul. 2001;20(3):255-64.

33. Jones AM, Im K-H, Savka MA, Wu M-J, DeWitt NG, Shillito R, Binns AN. Auxin-dependent cell expansion mediated by overexpressed auxin-binding protein 1. Science. 1998;282(5391):1114-7.

34. Borrego $E$, Kolomiets $M$. Synthesis and functions of jasmonates in maize. Plants. 2016;5(4):41.

35. Fernández-Calvo P, Chini A, Fernández-Barbero G, Chico JM, GimenezIbanez S, Geerinck J, Eeckhout D, Schweizer F, Godoy M, Franco-Zorrilla JM. The Arabidopsis bHLH transcription factors MYC3 and MYC4 are targets of JAZ repressors and act additively with MYC2 in the activation of jasmonate responses. Plant Cell. 2011;23(2):701-15.

36. Pauwels $L$, Goossens A. The JAZ proteins: a crucial interface in the jasmonate signaling cascade. Plant Cell. 2011;23(9):3089-100.

37. Srivastava AK, Orosa B, Singh P, Cummins I, Walsh C, Zhang C, Grant M, Roberts MR, Anand GS, Fitches E. SUMO suppresses the activity of the jasmonic acid receptor coronatine insensitive1. Plant Cell. 2018;30(9):2099-115.

38. Song S, Qi T, Huang H, Xie D. Regulation of stamen development by coordinated actions of jasmonate, auxin, and gibberellin in Arabidopsis. Mol Plant. 2013;6(4):1065-73.

39. Evans $\mathrm{NH}$. Modulation of guard cell plasma membrane potassium currents by methyl jasmonate. Plant Physiol. 2003;131(1):8-11.

40. Li N, Gügel IL, Giavalisco P, Zeisler V, Schreiber L, Soll J, Philippar K. FAXI, a novel membrane protein mediating plastid fatty acid export. PLOS Biol. 2015:13(2):e1002053

41. Wolf M, Cull IM, Helm J, Zuber M. Measuring thickness of excised mature corn pericarp 1. Agron J. 1969:61(5):777-9.

42. Knapp SJ, Stroup WW, Ross WM. Exact confidence intervals for heritability on a progeny mean basis. Crop Sci. 1985:25:192-4.

43. Saghai-Maroof MA, Soliman KM, Jorgensen RA, Allard R. Ribosomal DNA spacer-length polymorphisms in barley: mendelian inheritance, chromosomal location, and population dynamics. P Natl Acad Sci USA. 1984;81(24):8014-8.

44. Zhang J, Zhang Q, Cheng T, Yang W, Pan H, Zhong J, Huang L, Liu E. Highdensity genetic map construction and identification of a locus controlling weeping trait in an ornamental woody plant (Prunus mume Sieb. et Zucc). DNA Res. 2015;22(3):183-91.

45. Li H, Durbin R. Fast and accurate short read alignment with BurrowsWheeler transform. Bioinformatics. 2009;25(14):1754-60

46. Huang X, Feng Q, Qian Q, Zhao Q, Wang L, Wang A, Guan J, Fan D, Weng $Q$, Huang T. High-throughput genotyping by whole-genome resequencing. Genome Res. 2009:19(6):1068-76.

47. Liu D, Ma C, Hong W, Huang L, Liu M, Liu H, Zeng H, Deng D, Xin H, Song J. Construction and analysis of high-density linkage map using highthroughput sequencing data. PLoS ONE. 2014;9(6):e98855. 
48. Van Os H, Stam P, Visser RG, Van Eck HJ. SMOOTH: a statistical method for successful removal of genotyping errors from high-density genetic linkage data. Theor Appl Genet. 2005;112(1):187-94.

49. Xu S, Hu Z. Mapping quantitative trait loci using distorted markers. Int J Plant Genomics. 2009;2009:410825.

50. Kosambi DD. The estimation of map distances from recombination values. Ann Eugen. 1943;12:172-5.

51. Yang J, Hu C, Hu H, Yu R, Xia Z, Ye X, Zhu J. QTLNetwork: mapping and visualizing genetic architecture of complex traits in experimental populations. Bioinformatics. 2008, 24:721-3.

52. Churchill GA, Doerge RW. Empirical threshold values for quantitative trait mapping. Genetics. 1994;138(3):963-71.

53. Stuber CW, Edwards M, Wendel JF. Molecular marker-facilitated investigations of quantitative trait loci in maize. II. factors influencing yield and its component traits 1. Crop Sci. 1987:27(4):639-48.

54. Livak KJ, Schmittgen TD. Analysis of relative gene expression data using real-time quantitative PCR and the $2^{-\Delta \Delta C T}$ method. Methods. 2001;25(4): 402-408.

\section{Publisher's Note}

Springer Nature remains neutral with regard to jurisdictional claims in published maps and institutional affiliations.

Ready to submit your research? Choose BMC and benefit from:

- fast, convenient online submission

- thorough peer review by experienced researchers in your field

- rapid publication on acceptance

- support for research data, including large and complex data types

- gold Open Access which fosters wider collaboration and increased citations

- maximum visibility for your research: over $100 \mathrm{M}$ website views per year

At BMC, research is always in progress.

Learn more biomedcentral.com/submissions 